\title{
Contributions of Different Aeroacoustic Sources to Aircraft Cabin Noise
}

\author{
Nan Hu, ${ }^{*}$ Heino Buchholz, and Michaela Herr \\ German Aerospace Center (DLR), Lilienthalplatz 7, D-38108 Braunschweig, Germany \\ Carsten Spehr and Stefan Haxter \\ German Aerospace Center (DLR), Bunsenstraße 10, D-37073 Göttingen, Germany
}

\begin{abstract}
The turbulent boundary layer (TBL) on the fuselage, jet noise and the air condi1 tioning system (ACS) are considered as three important aeroacoustic sources of aircraft cabin noise. To improve current cabin noise prediction approaches as well as to investigate the different noise sources and their respective noise transfer paths, flight tests with DLR's A320-232 research aircraft 'D-ATRA' were carried out within the German national (LuFo IV) project SIMKAB. Extensive measurement data were collected using microphones inside the cabin, unsteady surface-pressure sensors for the characterization of the external TBL- and jet noise induced fuselage excitation, and accelerometers mounted at the frame structure, fuselage skin fields and cabin panels. Flight speed and -level as well as engine and air conditioning system operating conditions were varied to separately evaluate their parametric effects on cabin noise. The analysis of this extensive data base is still ongoing; in the current paper the focus is set on the results from microphone measurements at various longitudinal positions inside the cabin. Both TBL- and jet noise induced contributions increase towards the rear, reflecting the natural growth of the TBL thickness and typical jet noise radiation characteristics. Contrary to that the air conditioning system noise is of minor importance.
\end{abstract}

\section{Nomenclature}

FL

Kinematic viscosity $\left[\mathrm{m}^{2} / \mathrm{s}\right]$ Power spectral density of sound/surface pressure fluctuations $\left[\mathrm{Pa}^{2} / \mathrm{Hz}\right]$ Air density $\left[\mathrm{kg} / \mathrm{m}^{3}\right]$ Shear stress at the wall $[\mathrm{Pa}]$ Skin friction coefficient [-] Mach number [-] Dynamic pressure $[\mathrm{Pa}]$ Reynolds number [-] Flow velocity $[\mathrm{m} / \mathrm{s}]$ Friction velocity $[\mathrm{m} / \mathrm{s}]$ Air conditioning system Flight level $[100 \mathrm{ft}]$

OASPL A-weighted overall sound/surface pressure level [dB(A)]

SPL

TAS

TBL A-weighted 1/3-octave band sound pressure level $[\mathrm{dB}(\mathrm{A})]$ True air speed $[\mathrm{m} / \mathrm{s}]$ Turbulent boundary layer

\footnotetext{
${ }^{*}$ Research Engineer, Department of Technical Acoustics, Institute of Aerodynamics and Flow Technology, German Aerospace Center (DLR), nan.hu@dlr.de
} 


\section{Background and Motivation}

Airline passenger comfort has become an important aspect in the context of new aircraft designs. Cabin noise belongs to one of the deciding conditions in this sense. ${ }^{1}$ In the past years pressure fluctuations underneath TBLs have been extensively studied. Well-known prediction models such as the ones by Chase ${ }^{2}$ Corcos $^{3}$ or Goody ${ }^{4}$ were developed for zero pressure-gradient TBLs at low subsonic Mach numbers $M a$. At high subsonic $M a$, comparable to airplane cruise conditions, only limited research was performed. One wind-tunnel experiment under such conditions was reported by Ehrenfried and Koop. ${ }^{5}$ Efimtsov $^{6}$ derived an adapted Corcos model, covering also transonic $M a$. The characteristics of TBL-induced cabin noise have been studied by Graham ${ }^{7,8}$ based on the structural response of generic flat plate configurations. A well-documented theoretical overview of the TBL-induced excitation of flat plates and their corresponding vibroacoustic response behavior is provided by Blake. ${ }^{9}$

Jet noise is a second source of broadband pressure fluctuations on the fuselage outer skin. For typical transport aircraft its contribution is expected to become more significant in the aft region of the cabin. Research on the acoustic near field of a co-axial jet was published by Tinney and Jordan. ${ }^{10}$ In particular for rear-mounted engines (but to some extent also for wing-mounted engine configurations) the pressure amplitude and phase of the incident sound waves can be strongly modified by refraction and scattering due to the TBL. This effect was studied by Siefert et al. ${ }^{11}$ using computational aeroacoustic simulations.

To the authors' knowledge, there are no publications available regarding the effect of the ACS on cabin noise. Most of the past investigations based on flight tests concentrated on the induced pressure fluctuations from the TBL and jet noise on the fuselage outer skin. ${ }^{12-15}$ The corresponding structure vibration response was investigated by Wilby and Gloyna. ${ }^{16,17}$ Cabin noise reduction treatments were tested in flight by Bhat and Wilby, ${ }^{18}$ applying damping tapes and rubber wedges on the inner fuselage skin of a Boeing aircraft to modify the radiation features. So far, no flight test results have been published showing noise distributions inside the cabin over a large extent of the passenger area or on the detailed characteristics and relative ranking of the different sources.

The goal of this work is to identify and rank the different contributions to cabin noise through measurements of their respective longitudinal noise distributions inside the cabin for different flight configurations. Flight and operation conditions were selectively modified for a separate source assessment. In the following second part of the paper, the flight test setup and the different flight configurations are described. The results will be presented and discussed in the third part.

\section{Test Setup and Procedure}

Eleven pairs of 1/4-inch Brüel \& Kjær multi-field microphones (type 4961) were installed at the height of the seat headrests, in the middle of every second seat row on the left and in the aisle, respectively. Their plan view positions are sketched in figure 1.

Data were acquired at a sampling rate of $50 \mathrm{kHz}$ using a multi-channel measurement system installed in the front area of the airplane. To minimize the background noise caused by the measurement system thick mats of foam material were applied between the test area and the measurement system. Meansquared sound pressures at each longitudinal position were averaged between the seat and the aisle microphones, except for the last position (11), where the seat position microphone failed during the tests. Tonal peaks in the measured cabin noise spectra due to fan noise, hydraulic noise, or electrical disturbances were eliminated during data processing, because the focus is set on the three broadband aeroacoustic sources mentioned above.

The ACS operation modes are varied by changing the conditions of the pack flow and the cabin recirculation fans. The pack flow, regulated by the pack controller (which operates the respective control and bypass valves as well as the subsequent temperature-regulation by air-to-air heat exchangers), is the

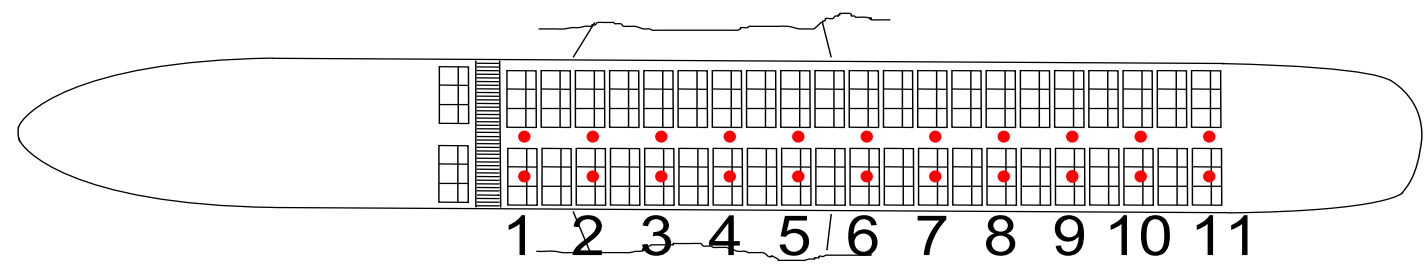

Figure 1: Sketch of the microphone positions inside the cabin. 
bleed air extracted from the engine compressor stage. The correspondingly regulated air from the two packs is then fed to a mixing unit (also connected to cabin air supplied by the recirculation fans) from where it is further distributed into the cabin and the cockpit. The recirculation fans switched on can accelerate the distribution process. Different ACS operation modes for two flight speeds in cruise at flight level (FL) 350, corresponding to a nominal altitude of $35000 \mathrm{ft}$ for standard atmospheric conditions, were selected as summarized in table 1.

Table 1: ACS operation modes (FL 350).

\begin{tabular}{|c|c|c|c|c|}
\hline $\boldsymbol{M a}[-]$ & TAS $[\mathbf{m} / \mathbf{s}]$ & Pack flow & Recirculation fans & Engine conditions \\
\hline 0.72 & 212 & Off & Off & Cruise \\
\hline 0.72 & 212 & Low & Off & Cruise/Idle/MaxCP \\
\hline 0.72 & 212 & Norm & On & Cruise \\
\hline 0.78 & 231 & Off & Off & Cruise \\
\hline 0.78 & 231 & Low & Off & Cruise \\
\hline
\end{tabular}

During all test flights the gaspers above the passenger seats were closed. Three different engine working conditions, i. e. cruise, flight idle, and maximum continuous power (MaxCP), were set at a constant low-noise ACS operation mode (pack flow at "low" and cabin recirculation fans at "off") to separate the noise contributions from the TBL and the jet. Additional three FLs were studied for this condition as surveyed in table 2 .

Table 2: Selected test conditions for constant ACS operation mode (low-off).

\begin{tabular}{|c|c|c|c|}
\hline Ma [-] & TAS [m/s] & FL $[\mathbf{1 0 0} \mathbf{f t}]$ & Engine conditions \\
\hline 0.70 & 214 & 270 & Cruise/Idle/MaxCP \\
\hline 0.77 & 237 & 270 & Cruise/Idle/MaxCP \\
\hline 0.71 & 213 & 310 & Cruise/Idle/MaxCP \\
\hline 0.78 & 236 & 310 & Cruise/Idle/MaxCP \\
\hline 0.72 & 212 & 350 & Cruise/Idle/MaxCP \\
\hline 0.80 & 236 & 350 & Cruise/Idle/MaxCP \\
\hline 0.72 & 206 & 390 & Cruise/Idle/MaxCP \\
\hline 0.82 & 236 & 390 & Cruise/Idle/MaxCP \\
\hline
\end{tabular}

Ma numbers for different FLs were adapted to keep approximately constant true air speeds (TAS) for direct comparisons, accounting for the variations in sound speed with altitude. According to table 2 tests were performed at a "lower TAS" $(\approx 213 \mathrm{~m} / \mathrm{s})$ and "higher TAS" $(\approx 236 \mathrm{~m} / \mathrm{s})$, respectively. A larger deviation, when compared to the actual values at the other FLs, is documented for the lower TAS case at FL $390(206 \mathrm{~m} / \mathrm{s})$. With regard to its influence on sound pressure level this 3-\% deviation is considered acceptable.

The measurement time for cruise conditions was $60 \mathrm{~s}$. For idle and MaxCP engine conditions, i.e. decelerated or accelerated aircraft motion, mean-squared sound pressure time histories were extracted and averaged within ranges of approximately $M a \pm 0.01$ (for distinct $M a$ values in the tables), corresponding to a measurement time of about $10 \mathrm{~s}$. The herein shown sound pressure spectra and derived overall sound pressure levels (OASPLs) are all A-weighted. 


\section{Test Results}

\section{A. Air Conditioning System Noise}

If both the pack controller and cabin recirculation fans are switched off, ACS noise will be eliminated. This condition (ACS at "off-off") provides the reference in figure 2, showing the ACS-induced OASPL increase measured along the cabin for conditions as summarized in table 1 (only cruise). Overall, the noise increase of $<2 \mathrm{~dB}(\mathrm{~A})$ is comparatively small and restricts to the front region of the measurement area. Maximum noise increase is observed at microphone position 3 and for the standard operation mode "norm-on". Moreover, OASPL differences diminish with increasing $M a$ (data available for condition "low-off" only) which is the result of correspondingly increased TBL-induced broadband noise masking the otherwise unchanged ACS contribution at higher Ma (absolute noise data not shown herein).

In view of these overall results it should be recalled that all gaspers were closed during the test, i. e. the cabin was vented by air outlets located between the hat racks and ceiling panels. The influence of ACS noise might further increase if these individual air outlets are in use.

In figures 3 to 5 a more detailed analysis on the measured ACS noise maxima and corresponding source localization is provided. Figure 3 presents the differences in $1 / 3$-octave band sound pressure spectra at microphone position 3 for the cases shown in figure 2. A strong SPL increase amounting up to more than $8 \mathrm{~dB}$ is found at frequencies around $3 \mathrm{kHz}$, representing the main reason for the shown $\triangle$ OASPL maximum along the cabin, if the ACS is turned on. A narrowband spectral analysis proved that the shown peaks are not tonal but broadband in nature. Their frequency location is independent of the TAS. Note that such $\triangle$ SPL peaks are not observable in the rear region, where the ACS leads to a noise increase of only about $1-2 \mathrm{~dB}$ at frequencies above $2 \mathrm{kHz}$ (not shown). Accordingly, the resulting impact on the A-weighted OASPL, as documented in figure 2, is negligible.

Figure 4 shows differences in 1/3-octave band acceleration spectra caused by the ACS in the standard mode "norm-on" (again, relative to the condition "off-off"), as measured on the cabin panels and the passenger floor at $M a=0.72$. An acceleration level increase of more than $15 \mathrm{~dB}$ around $3 \mathrm{kHz}$ is measured on the passenger floor in the region underneath microphone position 3, whereas a level increase of less than $5 \mathrm{~dB}$ is measured on the side-wall panel and the ceiling panel in this region. This suggests that the documented ACS noise increase around $3 \mathrm{kHz}$ comes from underneath the passenger floor. The packs

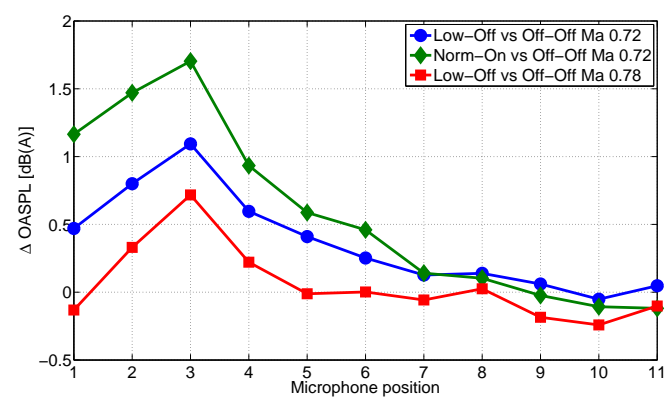

Figure 2: Differences in OASPL with and without working ACS in cruise at FL 350.

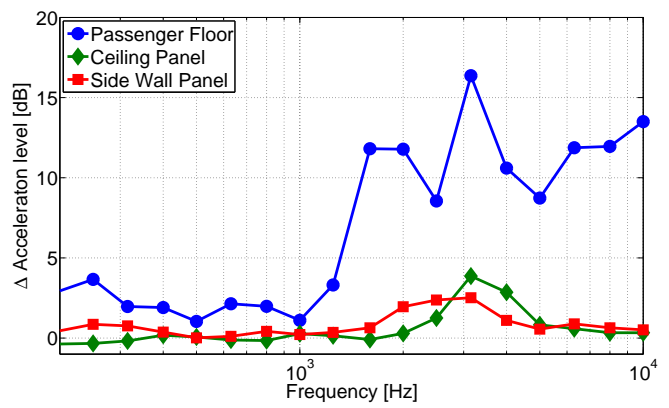

Figure 4: Differences in 1/3-octave band acceleration spectra between working ACS operation modes at "norm-on" and at "off-off" in cruise.

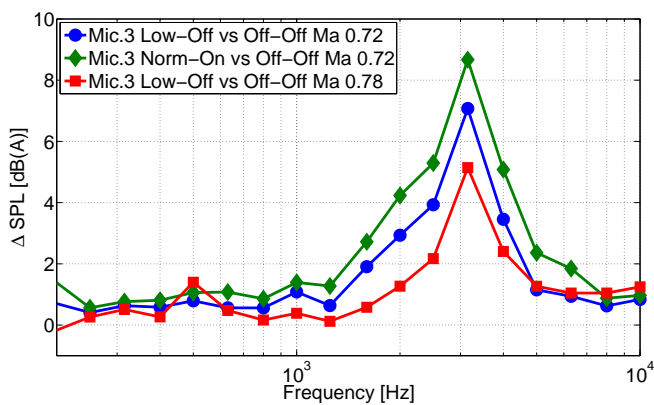

Figure 3: Differences in 1/3-octave band sound pressure spectra with and without working ACS in cruise (microphone position 3).

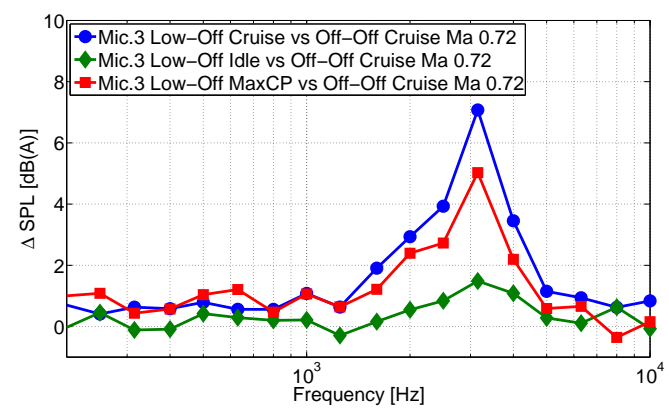

Figure 5: Differences in 1/3-octave band sound pressure spectra for different engine working conditions with and without working ACS. 
(air generation unit) are located in this region and are assumed to be the cause of the noise and vibration increase.

This assumption is further supported by figure 5 , presenting the differences in $1 / 3$-octave band sound pressure spectra caused by the ACS in the operation mode "low-off" for different engine conditions. Accordingly, ACS noise is affected by the engine operating condition related to variations in the amount of bleed air provided. Almost no SPL increase is measured for the idle case, where the maximum volume flow rate of the bleed air to the packs is reduced. The maximum SPL increase at $3 \mathrm{kHz}$ (of about $7 \mathrm{~dB}$ ) is measured in cruise, while for the MaxCP case maximum $\triangle \mathrm{SPL}$ is about $2 \mathrm{~dB}$ smaller. The observed noise reduction in this case might be the result of a reduced pack flow, attributed to a higher power requirement of the engine.

\section{B. TBL Noise}

For the following analysis it is supposed that for the idle cases the TBL-induced cabin noise dominates the remaining engine noise related contributions. The comparison of TBL- and jet-induced interior noise shown in the next section confirms this assumption at least for the lower FLs, i. e. for FL 270 and FL 310. Supplemented by the above results, that ACS noise is of minor importance for this test airplane, it will be accordingly assumed that the measured cabin noise for the idle case is solely due to TBL-induced pressure fluctuations on the fuselage.

Usually, power spectral densities $\phi_{p}$ of TBL-induced wall-pressure fluctuations are scaled based on TBL outer variables as $\phi_{p} \propto q^{2} \delta / u$, or based on mixed variables as $\phi_{p} \propto \tau_{w}^{2} \delta / u$, where $q=\frac{1}{2} \rho u^{2}$, and $\tau_{w}=\frac{1}{2} \rho u^{2} c_{f}$. Accordingly normalized surface pressure spectra generally match better in the lower to mid frequency domain when the scaling is based on outer or mixed variables, whereas scaling based on pure inner variables, i. e. according to $\phi_{p} \propto \tau_{w}^{2} \nu / u_{\tau}^{2}$, is suitable only at higher frequencies. With a simple flat plate TBL assumption, i. e. $\delta \propto x e^{-\frac{1}{5}}$ and $c_{f} \propto R e^{-\frac{1}{5}}$ for large $R e$, one obtains the following simplified relationships between wall-pressure power spectral densities (fuselage excitation) and the flow conditions:

$$
\begin{array}{ll}
\phi_{p} \propto q^{2} \delta / u & \rightarrow \phi_{p} \propto \rho^{2} u^{2.8} x^{0.8} \nu^{0.2} \quad \text { (outer scaling), or } \\
\phi_{p} \propto \tau_{w}^{2} \delta / u & \rightarrow \phi_{p} \propto \rho^{2} u^{2.4} x^{0.4} \nu^{0.6} \quad \text { (mixed scaling) }
\end{array}
$$

Table 3 summarizes the flight test conditions for two microphone positions, the lower and higher TAS cases, and for different FLs that have been selected for the parametrical analysis conducted herein. It is worth mentioning that the underlying scaling assumption of the Efimtsov model, ${ }^{6}$ covering transonic $M a$ numbers, is $\phi_{p} \propto \rho^{2} u_{\tau}^{3} \delta$. This leads to a relationship $\phi_{p} \propto \rho^{2} u^{2.5} x^{0.5} \nu^{0.5}$ which provides predicted trends between the two bracketing relationships selected above.

Eqs. 1 and 2 provide rough estimates for the scaling behavior of the TBL-induced excitation of the fuselage. In the following these will be directly compared with the corresponding tendencies measured inside the cabin, i. e. observed differences in the scaling behavior of mean-squared surface and -sound pressures are expected to give a first impression of the relevant fuselage/frame transfer characteristics. Depending on the context, the "S" in OASPL will have to be read as "Sound" or "Surface", respectively.

Figure 6 shows the OASPL differences at microphone position 2 for FL 310, 350 and 390 relative to FL 270. Accordingly, similar tendencies as predicted for the fuselage excitation are also measured inside the cabin. The OASPL decreases with increasing FL, because the air density is significantly reduced at the higher FLs. The OASPL difference between FL 270 and FL 390 amounts about $4 \mathrm{~dB}(\mathrm{~A})$ for both the lower and higher TAS. For all selected cases the estimated decrease in surface pressure power spectral density is about $1 \pm 0.5 \mathrm{~dB}$ lower than the measured decrease in sound power inside the cabin, however, the observed agreement is still remarkably close.

Table 3: Flight conditions and environmental parameters.

\begin{tabular}{|l|c|c|c|c|}
\hline & TAS $=\boldsymbol{u}[\mathbf{m} / \mathbf{s}]$ & $\boldsymbol{x}, \mathbf{m i c}$. pos. 2/10 $[\mathbf{m}]$ & $\boldsymbol{\rho}\left[\mathrm{kg} / \mathbf{m}^{\mathbf{3}}\right]$ & $\boldsymbol{\nu}\left[\mathbf{m}^{\mathbf{2}} / \mathbf{s}\right]$ \\
\hline FL 270 & $214 / 237$ & $13 / 25.4$ & 0.50 & $3.1^{*} 10^{-5}$ \\
\hline FL 310 & $213 / 236$ & $13 / 25.4$ & 0.44 & $3.4^{*} 10^{-5}$ \\
\hline FL 350 & $212 / 236$ & $13 / 25.4$ & 0.38 & $3.8^{*} 10^{-5}$ \\
\hline FL 390 & $206 / 236$ & $13 / 25.4$ & 0.32 & $4.3^{*} 10^{-5}$ \\
\hline
\end{tabular}




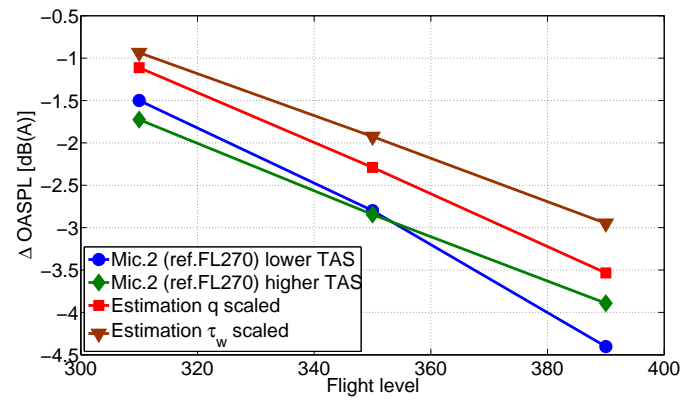

Figure 6: Differences in OASPL for different FLs relative to FL 270 in the idle case.

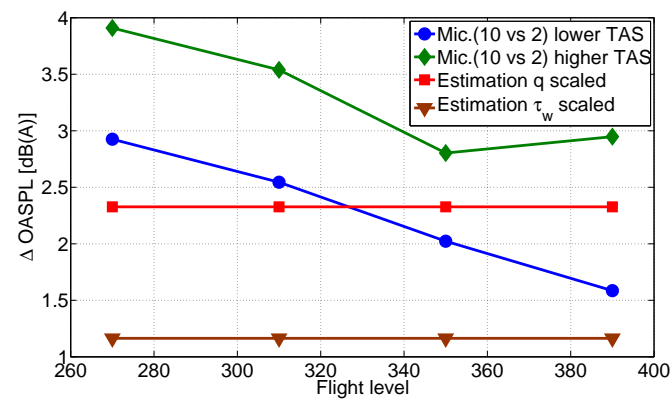

Figure 7: Differences in OASPL between microphone 10 and 2 for different FLs in the idle case.

The effect of the TBL growth from the front towards the rear fuselage is elaborated in figure 7 , showing the measured OASPL increase between microphone positions 2 and 10. Cabin noise measurement data for the four FLs and the lower and higher TAS are compared to the corresponding estimates of the fuselage surface pressure fluctuations. Overall, the surface pressure estimate based on TBL outer variables $\left(\phi_{p} \propto q^{2} \delta / u\right)$, as preferably applied for non-zero pressure gradient TBLs, comes closer to the actually measured cabin noise OASPL differences. Note that the measured $\triangle$ OASPLs show a dependence on FL and TAS, whereas the estimates stay at constant values expressed by $\phi_{p} \propto x^{0.8}$ or $\phi_{p} \propto x^{0.4}$, respectively (because the only altered variable between measurements at positions 2 and 10 for otherwise constant ambient conditions is the longitudinal position $x$, determining the TBL parameters $\delta$ and $c_{f}$ ). Larger $\triangle$ OASPLs are measured at the higher TAS and with decreasing FL. However, this trend is not perfectly kept for the higher TAS at FL 390, where it is believed that engine noise might not be negligible.

The observed discrepancies between predicted trends for the fuselage excitation and the measured trends inside the cabin can not be fully clarified based on the current test data. Unsteady surface pressure sensor data are so far not available for positions at the rear fuselage, and TBL profiles were not measured in flight. Therefore, the question has to be left open, whether discrepancies are due to transfer specifics of the fuselage/structure or simply to inadequacy of the flat-plate TBL assumptions. In this respect it is planned to conduct supplementing CFD simulations to derive the lacking local flow parameters ( $\delta$ and $c_{f}$ ), particularly to quantify actual variations of the $\delta$ ratios in dependence of the FL and TAS.

The velocity scaling behavior of mean-squared interior sound and fuselage surface pressures is discussed in the following. Figure 8 shows the respective OASPL differences between the higher and lower TAS at one selected longitudinal position $x=13 \mathrm{~m}$ (position 2), where also unsteady surface pressure data were acquired. ${ }^{\mathrm{a}}$ It is noticeable that the OASPL differences measured inside the cabin amount $3-4 \mathrm{~dB}(\mathrm{~A})$ for an increase of only $10 \%$ in velocity. I. e. cabin noise $\triangle$ OASPLs are about $2-3 \mathrm{~dB}(\mathrm{~A})$ larger than the measured (except for FL $390^{\mathrm{b}}$ ) and predicted differences in corresponding surface pressure levels. The derived velocity scaling power law for TBL-induced interior noise would accordingly read $\phi_{p} \propto u^{7-9}$ (compared to $\phi_{p} \propto u^{2.4-2.8}$ for the excitation). Note that individual velocity exponents are frequency-dependent, as will be shown in the following.

One possible explanation for the observed behavior is seen in a velocity-dependent transfer efficiency. In Figure 9 FL 270 is selected for a more detailed analysis of the obtained level differences between the higher and lower TAS in the frequency domain. Differences between 1/3-octave band spectra for the lower and higher TAS are herein plotted for the TBL-induced cabin noise- (blue curve), and surface pressure (green curve) spectra. ${ }^{c}$ Moreover, also the TAS-induced differences in the "local transfer efficiency", herein defined as the difference between the measured cabin noise- and unsteady surface pressure spectra at a given $x$-position, are evaluated (red curve).

The blue curve indicates that the documented $\Delta$ OASPL in figure 8 is mainly related to a $\Delta$ SPL increase of TBL-induced cabin noise between $800 \mathrm{~Hz}$ and $3 \mathrm{kHz}$. The maximum increase at frequencies around $2 \mathrm{kHz}$ amounts about $6 \mathrm{~dB}$. In contrast, the TBL pressure fluctuations show a relative constant difference of order $1 \mathrm{~dB}$ over the whole frequency domain, which is according to expectation. Therefore,

\footnotetext{
a 30 unsteady surface pressure sensors were installed on 3 dummy windows located in the front part of the measurement area. The shown results in figures 8, 9, and 11 were obtained by averaging over all sensors on the window closest to position 2 (11 sensors in total). For more details of the used setup the reader might refer to Haxter and Spehr. ${ }^{15}$

${ }^{b}$ The unsteady surface pressure measurement result at FL 390, deviating from the predictions by roughly 1 dB must not be overrated, given an estimated error band of at least $\pm 1 \mathrm{~dB}$ for surface pressure differences between repetitive test flights.

${ }^{\mathrm{c}} \mathrm{A}$ Strouhal-dependence of TBL-induced hydrodynamic surface pressure spectra can be neglected herein because the frequency-shift caused by a $10 \%$-velocity increase will not significantly express in $1 / 3$-octave band spectra.
} 


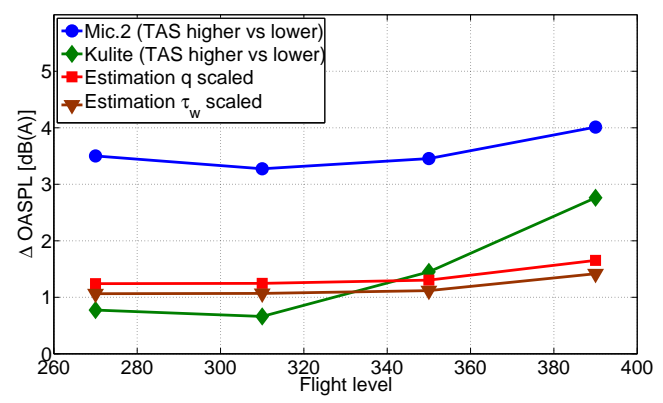

Figure 8: Differences in OASPL between the higher and lower TAS for different FLs in the idle case $(x=13 \mathrm{~m})$.

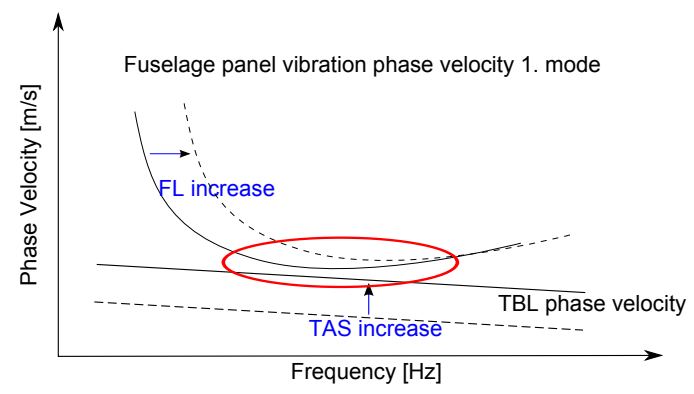

Figure 10: Schematic of phase velocity trends according to Wilby and Gloyna. ${ }^{16}$

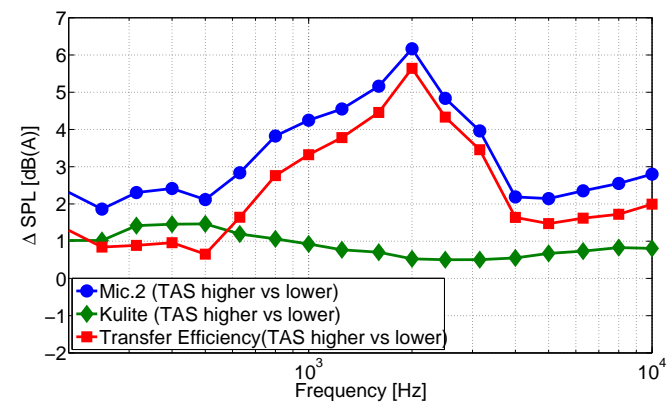

Figure 9: Differences in 1/3-octave band sound pressure spectra between the higher and the lower TAS at FL 270 in the idle case $(x=13 \mathrm{~m})$.

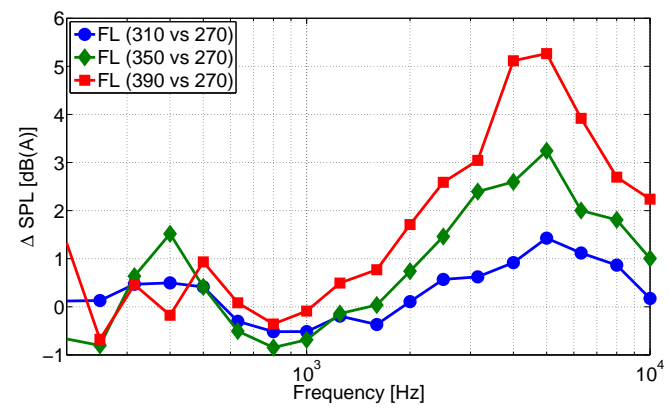

Figure 11: Differences in 1/3-octave band transfer efficiency spectra for different FLs relative to FL 270 (higher TAS, idle, $x=13 \mathrm{~m}$ ).

the derived TAS-induced increase of the transfer efficiency features a similar spectral shape as the cabin noise $1 / 3$-octave band difference spectra.

Both the velocity- and frequency-dependence of the transfer efficiency might be explained by coincidence effects induced by phase velocity matching: It is assumed that for the higher TAS the phase velocity of the TBL pressure fluctuations on the fuselage comes close to the vibration phase velocity of the fuselage panels within certain frequency bands, leading to an increased transfer efficiency in this range.

This phenomenon is tentatively illustrated in figure 10. Schematic phase velocity trends for the fuselage panel vibration and the TBL pressure fluctuations are extracted from the work of Wilby and Gloyna. ${ }^{16}$ The underlying measurement results show that the fuselage panel vibration is dominated by the first mode (one-half wavelength between the stringers) in a certain frequency domain. The shown curve is different from the vibration characteristics of an infinitely large plate $(u \propto \sqrt{f})$, and mainly depends on the circumferential distance between two stringers. Contrary to that, the phase velocity of TBL pressure fluctuations decreases slightly towards higher frequencies. Generally, when the phase velocity between the flow excitation and the fuselage panel are closer, the transmission will be more efficient. In the case of equal phase velocities, hydrodynamic coincidence between the flow and the structure leads to a very efficient power transmission. As the flight speed increases, also the TBL pressure fluctuation phase velocity increases and hence, comes closer to the phase velocity of fuselage panels. Especially in the frequency domain close to a coincidence the increase in transfer efficiency will be more significant. Consequently, a larger SPL difference will be measured in the cabin at the respective frequencies. These expressed presumptions are also supported by measurements of Bhat and Wilby. ${ }^{18}$ Similar to the observations made herein comparable trends for a velocity-induced increase of the transfer efficiency were measured.

Figure 11 has to be regarded in the same context. Here, the differences in transfer efficiency induced by different flight levels relative to FL 270 are shown at the higher TAS. An increasing transfer efficiency is found between $3 \mathrm{kHz}$ and $8 \mathrm{kHz}$ for increasing FLs. It is obvious that the fuselage panel strain can impact its vibration characteristics. For a higher cabin and ambient pressure difference, the fuselage panel strain increases which leads to a higher vibration phase velocity. Wilby and Gloyna ${ }^{16}$ show that the curve of the first fuselage panel vibration mode is then shifted to a higher frequency domain (Figure 10). Also 
a temperature difference might impact the fuselage panel vibration in the same way. The recorded flight test information data documents that the cabin and ambient pressure differences were kept relatively constant for different FLs, whereas the temperature dropped by about $10^{\circ} \mathrm{C}$ by each FL increase.

\section{Jet Noise}

Jet-induced interior noise can be isolated for the cruise and MaxCP cases by subtracting the TBL-induced noise contributions, herein represented by the idle case. For microphone positions upstream of position 5 (located close to the wing root trailing-edge) $|\Delta \mathrm{OASPL}| \ll 1 \mathrm{~dB}$ was measured relative to the idle case, i. e. jet-induced contributions are buried within TBL-induced noise and can be neglected in the front part of the cabin. The same holds for the lower TAS at the lower FLs (FL 270 and FL 310) for all measurement positions in cruise. Therefore, jet-induced noise could not be extracted for those cases.

Results from the energetic subtraction are presented in figure 12 for FL 350. Herein, jet-induced cabin noise is plotted in polar coordinates with the center of the engine nozzle exit taken as origin. ${ }^{\mathrm{d}}$ Figure 12 (a) shows the derived uncorrected "geometric directivity", while figure 12 (b) presents the equivalent source "radiation directivity" referred to the retarded source position at emission time, accounting for the forward motion of the aircraft. The latter representation format allows the direct comparison with typical isolated engine noise directivities with the particularity that the shown modulated source directivity also incorporates installation effects (like sound shielding), and effects induced by the sound transfer through the TBL (refraction and scattering of sound waves) as well as through the fuselage/frame structure into the cabin.

Interestingly, the accordingly extracted interior noise data show a very good qualitative agreement with published jet noise directivities, e. g. the measurement data for a co-axial jet engine by Tinney and Jordan. ${ }^{10}$ Maximum radiation lies in the angle range between $20^{\circ}-40^{\circ}$ which holds for all engine working conditions under consideration. Future work on this subject will focus on more detailed comparisons

\footnotetext{
${ }^{\mathrm{d}}$ If one assumes jet noise as a point source located at the nozzle exit, the sound radiation angle can be calculated and also the SPL can be corrected for the different radiation distances to each microphone position. Strictly speaking, jet noise cannot be represented by a simple point source. Particularly, the assumed farfield condition does not hold for the lower frequencies included in the OASPL integration.
}

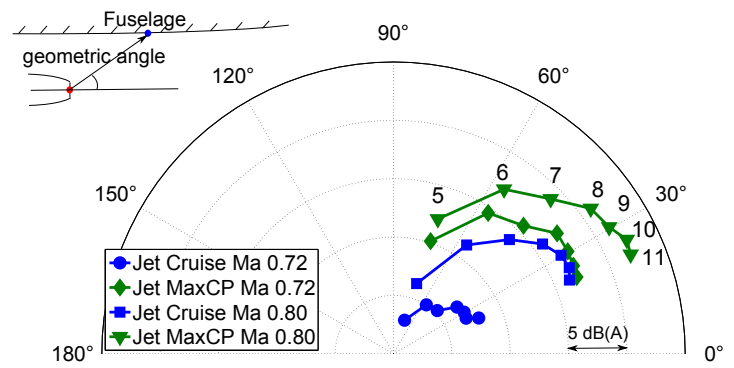

(a) Geometric directivity.

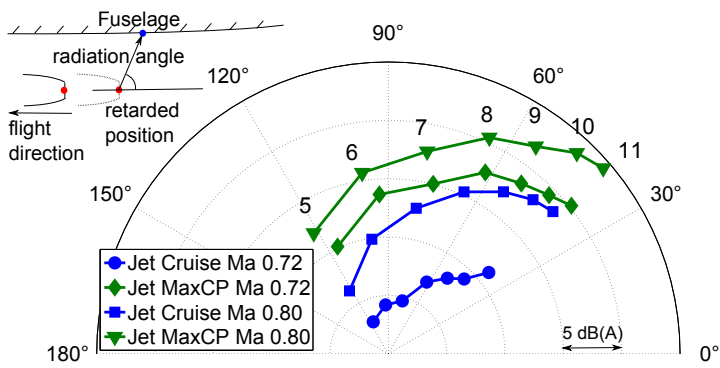

(b) Radiation directivity.

Figure 12: Relative OASPL of jet-induced interior noise in polar coordinates at FL 350.

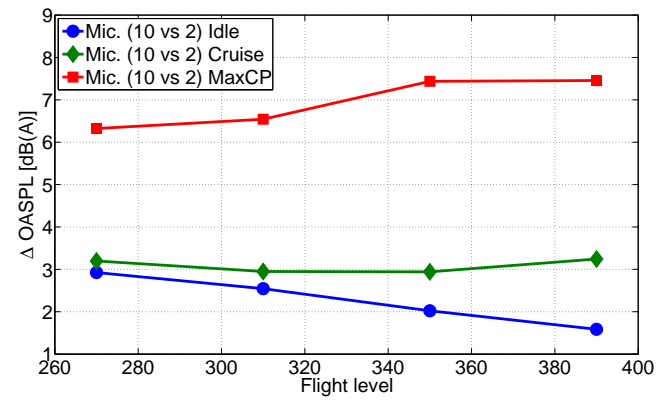

(a) Lower TAS.

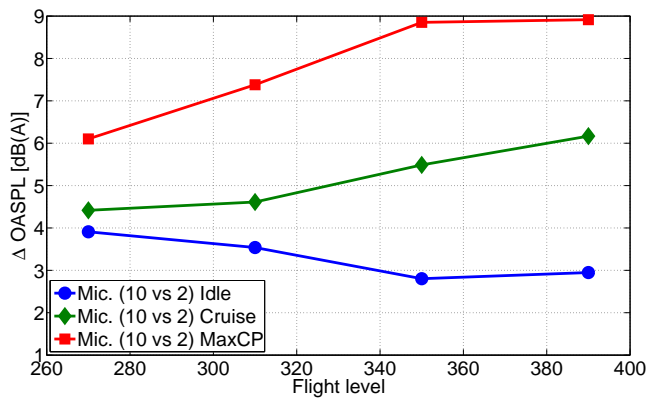

(b) Higher TAS.

Figure 13: Differences in OASPL between microphones 10 and 2 for different FLs and engine working conditions. 
of the derived radiation characteristics (with view from the cabin interior) with corresponding source characteristics of the V2500 'D-ATRA'-engine. In particular, CAA (computational aeroacoustics) simulations of both the isolated and installed engine with and without TBL-influence will be finally compared with the present test data. These comparisons will also include a separate assessment of the jet-noise induced unsteady surface pressures measured at the rear fuselage during additional 'D-ATRA' flight tests which are currently being post-processed. Based on the current state of data analysis, however, only a qualitative interior noise assessment of jet-related contributions can be provided.

Finally, the relative importance of jet noise- compared to TBL-related contributions can be roughly deduced from figure 13. The same data representation format as in figure 7 is applied, i. e. differences in A-weighted OASPLs between microphone positions 2 and 10 are shown for different engine working conditions and FLs at (a) the lower, and (b) the higher TAS. Correspondingly, the blue curves in figure 13 represent the noise increase due to the TBL development along the fuselage (data repeated from figure 7). New are the related OASPL differences for the cruise and the MaxCP cases. Following the discussion above, jet noise contributions are negligible for microphone position 2. Accordingly, the observed differences between the green or red curves (cruise or MaxCP cases), respectively, and the blue curves (idle cases) provide the OASPL increase at the rear (position 10) that is caused by jet noise.

As shown, the latter grows with increasing FL. To give an example, jet noise in cruise causes an OASPL increase at the rear cabin of about $0.5 \mathrm{~dB}(\mathrm{~A})$ at FL 270 for the higher TAS, while an OASPL increase of more than $3 \mathrm{~dB}(\mathrm{~A})$ is documented at FL 390. One reason for the growing relative importance of jet-related interior noise is seen in the reduction of TBL- induced noise with increasing FL (decreasing $\rho$ ), as has been discussed in the previous section. Moreover, in the cruise case jet-induced interior noise gains in importance as the TAS increases (not observed in the MaxCP case where jet-induced noise generally provides larger signal-to-noise ratios when referred to the TBL-induced "background noise"). For FL 390 an OASPL increase of about $1.5 \mathrm{~dB}(\mathrm{~A})$ is measured in cruise for the lower TAS, compared to more than $3 \mathrm{~dB}(\mathrm{~A})$ for the higher TAS. Note that a measured 3-dB increase relative to the idle case is tantamount to equal sound power of the TBL- and jet-induced noise in the rear cabin (position 10). Hence, at FL 350-390 jet-related noise in cruise, as measured at position 10, is of approximately the same order as TBL noise at the higher TAS. A contrario, at the lower TAS in cruise TBL-induced noise at the rear is larger than jet-induced noise, as documented by differences of $\Delta$ OASPL of $<3 \mathrm{~dB}(\mathrm{~A})$. Overall, an enhanced role of jet noise is documented for the MaxCP case when compared to cruise.

\section{Conclusion and Outlook}

Airbus A320 flight tests at different flight speeds, flight levels, as well as at variant operating conditions of the engines and the air conditioning system were conducted within the German national (LuFo IV) project SIMKAB. The used parametric approach enables the separate assessment of the different contributions to cabin noise. Herein, the broadband interior noise contributions due to the turbulent boundary-layer (TBL) excitation of the fuselage, jet noise and the air conditioning system (ACS) are considered.

According to the collected test data ACS noise plays a minor role. A noise increase of only about $1-2 \mathrm{~dB}(\mathrm{~A})$ in overall sound pressure level (OASPL) was measured in the front region of the test area if the ACS is switched on. The importance of the ACS noise is further reduced with increasing flight speeds. Due to their regulative effect on the ACS pack flow the engine working conditions also impact the measured ACS noise.

TBL-induced cabin noise OASPL increases by about 3-4 $\mathrm{dB}(\mathrm{A})$ when moving from the front measurement position (close to the wing root leading-edge) towards the rear of the cabin. Moreover, it rises with decreasing flight level and with increasing flight speed. Correspondingly extracted scaling dependencies have been compared with estimates and selected measurement results for the fuselage excitation. The derived transfer efficiency of the fuselage for TBL-induced pressure fluctuations was shown to increase between $800 \mathrm{~Hz}$ to $3 \mathrm{kHz}$ at higher flight speeds, leading to enhanced cabin noise contributions in this frequency range. TBL-induced noise is the dominant contributor in cruise for lower flight speeds at all tested flight levels (FL 270-390) and also for higher flight speeds at lower flight levels (FL 270 -310).

Jet noise affects mostly the rear part of the cabin. Its relative importance grows with increasing flight levels and with increasing flight speeds (the latter only for cruise condition). Jet-induced noise in cruise, as measured in the rear cabin, becomes of equal order as TBL-induced noise at higher flight levels (FL 350-390). Interestingly, the measured directional dependence of jet-noise related cabin noise contributions shows a remarkably good agreement with published measurement results of typical jet noise radiation directivities.

Future work of the authors on this extensive flight test data base will focus on the analysis of 
measured surface pressure fluctuations on the fuselage to further precise the excitation induced by both the TBL and jet-noise. These data will be particularly used for the validation and further-development of DLR's CAA methodologies. Related ongoing CAA activity include the simulation of fuselage surface pressure fluctuations due to both the transonic TBL, and to jet noise contributions, accounting for sound refraction and scattering within the TBL. The exact description and subsequent modeling of the excitation is considered a prerequisite for a final assessment of the transfer mechanisms which determine the herein drafted cabin noise characteristics.

\section{Acknowledgments}

The authors would like to thank the following institutions:

- The Federal Ministry of Economics and Technology (BMWi) for the financial support of the SIMKAB project as part of the German national aerospace research program (LuFo IV).

- Airbus Operations $\mathrm{GmbH}$ for the provided infrastructure and valuable assistance in test preparation and instrumentation of the 'D-ATRA' A320-232 test aircraft.

\section{References}

${ }^{1}$ Mellert, V., B. I. and Freese, N., "Impact of Sound and Vibration on Health, Travel Comfort and Performance of Flight Attendants and Pilots," Aerosol Science and Technology, Vol. 12, 2008, pp. 18-25.

${ }^{2}$ Chase, D., "Modeling the Wavevector-Frequency Spectrum of Turbulent Boundary Layer Wall Pressure," Journal of Sound and Vibration, Vol. 70(1), 1980, pp. 29-67.

${ }^{3}$ Corcos, G., "The Structure of the Turbulent Pressure Field in Boundary Layer Flows," Journal of Fluid Mechanics, Vol. 18, 1964, pp. 353-378.

${ }^{4}$ Goody, M., "Empirical Spectral Model of Surface Pressure Fluctuations," AIAA Journal, Vol. 42, No.9, 2004.

${ }^{5}$ Ehrenfried, K. and Koop, L., "Experimental Study of Pressure Fluctuations Beneath a Compressible Turbulent Boundary Layer," 14th AIAA/CEAS Aeroacuostics Conference, 2008.

${ }^{6}$ Efimtsov, B. M., "Characteristics of the Field of Turbulent Wall Pressure Fluctuations at Large Reynolds Numbers," Sov. Phys. Acoust., Vol. 28, No. 4, 1982, pp. 289-292.

${ }^{7}$ Graham, W., "Boundary Layer Induced Noise in Aircraft, Part I: The Flat Plate Model," Journal of Sound and Vibration, Vol. 192(1), 1996, pp. 101-120.

${ }^{8}$ Graham, W., "Boundary Layer Induced Noise in Aircraft, Part II: The Trimmed Flat Plate Model," Journal of Sound and Vibration, Vol. 192(1), 1996, pp. 121-138.

${ }^{9}$ Blake, W., Mechanics of Flow-Induced Sound and Vibration, Academic Press, INC., 1986.

${ }^{10}$ Tinney, C. and Jordan, P., "The Near Pressure Field of Co-Axial Subsonic Jets," Journal of Fluid Mechanics, Vol. 611, 2008, pp. 175-204.

${ }^{11}$ Siefert, M., Delfs, J. W., and Caruelle, B., "Refraction and Scattering in High Mach Number Boundary Layers," 17th AIAA/CEAS Aeroacoustics Conference, 2011.

${ }^{12}$ Bhat, W., "Flight Test Measurement of Exterior Turbulent Boundary Layer Pressure Fluctuations on Boeing 737 Airplane," Journal of Sound and Vibration, Vol. 14(4), 1971, pp. 439-457.

${ }^{13}$ Bhat, W., "Use of Correlation Technique for Estimating In-Flight Noise Radiated by Wing-Mounted Jet Engines on a Fuselage," Journal of Sound and Vibration, Vol. 17(3), 1971, pp. 349-355.

${ }^{14}$ Palumbo, D., "Determining Correlation and Coherence Lengths in Turbulent Boundary Layer Flight Data," Journal of Sound and Vibration, Vol. 331, 2012, pp. 3721-3737.

${ }^{15}$ Haxter, S. and Spehr, C., "Two-Dimensional Evaluation of Turbulent Boundary Layer Pressure Fluctuations at Cruise Flight Conditions," 18th AIAA/CEAS Aeroacoustics Conference, 2012.

${ }^{16}$ Wilby, J. and Gloyna, F., "Vibration Measurements of An Airplane Fuselage Structure, I Turbulent Boundary Layer Excitation," Journal of Sound and Vibration, Vol. 23(4), 1972, pp. 443-466.

${ }^{17}$ Wilby, J. and Gloyna, F., "Vibration Measurements of An Airplane Fuselage Structure, II Jet Noise Excitation," Journal of Sound and Vibration, Vol. 23(4), 1972, pp. 467-486.

${ }^{18}$ Bhat, W. and Wilby, J., "Interior Noise Radiated by an Airplane Fuselage Subjected to Turbulent Layer Excitation and Evaluation of Noise Reduction Treatments," Journal of Sound and Vibration, Vol. 18(4), 1971, pp. 449-464. 